\title{
Wood fingerprint recognition using knot neighborhood $K$-plet descriptors
}

\author{
Tobias Pahlberg • Erik Johansson • Olle Hagman • \\ Matthew Thurley
}

Received: March 28, 2014 / Accepted: October 4, 2014

\begin{abstract}
In the wood industry there is a wish to recognize and track wood products through production chains. Traceability would facilitate improved process control and extraction of quality measures of various production steps. In this paper, a novel wood surface recognition system that uses scale- and rotationally invariant feature descriptors called K-plets is described and evaluated. The idea behind these descriptors is to use information of how knots are positioned in relation to each other. The performance and robustness of the proposed system is tested on 212 wood panel images with varying levels of normally distributed errors applied to the knot positions. The results showed that the proposed method is able to successfully identify 99-100\% of all panel images with knot positional error levels that can be expected in practical applications.
\end{abstract}

Keywords Wood fingerprint, K-plet, knot neighborhood descriptor, object identification

\section{Introduction}

The motivation behind this work comes from companies wanting to recognize and track solid wood products through their production chain. The recognition method should not interfere with, or have a negative effect on the existing processes. The method should preferably utilize sensors already available in the production chain and not introduce new ones. Today, recognition and tracking tasks are often carried out by aid of barcode labels or paint (Uusijärvi 2000, p. 64-73; Dykstra et al. 2002, Flodin 2009).

This work is part of the EU project Hol-i-Wood Patching Robot, where one task is to recognize shuttering panels at an automatic patching station. The project will in the end have resulted in a completely automated patching system which will be placed at the wood products producer LIP-BLED in Slovenia.

Tracking capabilities of wood pieces in a non-invasive way requires a method to assign wood fingerprints to every "individual". A fingerprint should include properties such that it becomes

Tobias Pahlberg

Luleå University of Technology, Forskargatan 1, 93187, Skellefteå, Sweden

Tel.: +46-910-585336

E-mail: tobias.pahlberg@ltu.se

Erik Johansson

Luleå University of Technology, Forskargatan 1, 93187, Skellefteå, Sweden

SP Technical Research Institute of Sweden, Laboratorgränd 2, 93177 Skellefteå, Sweden

Tel.: +46-10-5166278

E-mail: erik.johansson@ltu.se 
possible to identify a unique wood piece. Visually salient features, geometric features or material properties are examples which can be utilized for this purpose.

The two most common, distinct, visual features on sawn lumber are often knots and growth ring patterns. However, the presence of visual features in the images, of course, highly depends on wood species, sawing method and image acquiring technique.

A robust matching method that does not rely on the wood piece keeping its exact shape is desirable since it will increase the number of possible applications. Wood products are often cut or milled into smaller pieces in industrial processes with rip-sawing, cross-cutting and other more product specific processes as examples. In these cases it would not be possible to directly use knot coordinates as measures of the positions of the features. Intrinsic geometrical relations between knots may be used instead.

In some applications it is advantageous if the recognition method can handle the case when an object appears at a different rotation or in a different scale. The products may not arrive at the camera station with a known orientation, or maybe the vision system is mounted on a mobile robot. In some cases it may be difficult to obtain the exact same camera setup and conditions in two places. Sometimes, it might not be possible or even desirable to have two similar camera setups due to high costs, space or production speed.

The objective of this study is to evaluate the performance and robustness of a novel matching algorithm for wooden panels. The proposed algorithm uses a feature descriptor called K-plets, originally proposed by Chikkerur et al. (2005) to encode the local neighborhoods of human fingerprint features (minutiae). Our paper was inspired by this original work, but here the $K$-plets are based upon knots and their relative positions. Using knot patterns when identifying wood surfaces has, to our knowledge, never been done before.

The proposed algorithm is not affected by different scales or rotations of the panel images, i.e., it is scale- and rotationally invariant. However, it is sensitive to other anisotropic geometric distortions. This approach would in a real-world application require a method for segmenting knots from clearwood. Since knot segmentation is well established in the literature (Astrand 1996, Funck et al. 2003; Todoroki et al. 2010), it is outside the scope of this article. Instead, the effect of the precision of knot segmentation is evaluated.

\section{Related work}

\subsection{Point pattern matching}

Points or regions in an image, which have a high probability of being detected even after geometric and radiometric distortions have been applied to the image, are usually referred to as feature points. Feature points could be, for example, distinctive blobs, corners or other high curvature points. If this is applied to wood, feature points could for example be knots, resin pockets and certain annual ring patterns. Except for naturally occurring features on wood surfaces, there may also be other features induced by handling, such as dirt or dents. Point pattern matching is a problem that consists of finding correct point matches between two images or scenes (Chang et al. 1997). This problem has previously been addressed in, for example, star and constellation recognition (Spratling and Mortari 2009), biometrics (Jain et al. 2007, Chap. 1-2), image stitching (Brown and Lowe 2007) and 3D point cloud registration (Gruen and Akca 2005).

According to Chang et al. (1997) there are a few problems associated with point pattern matching in general:

1. The images of the objects to be recognized can have been acquired using different camera setups and at different times. Hence, the objects can have different rotation, scale or position.

2. Due to radiometric differences and dissimilar noise conditions there may be inaccuracies introduced in the positions of the extracted feature points. 

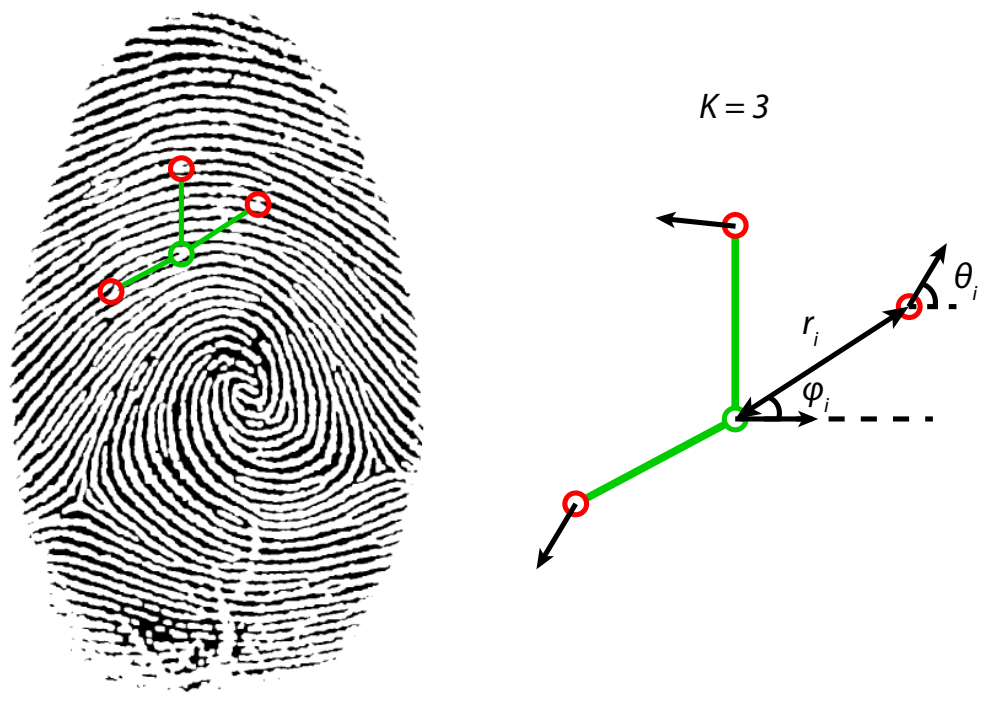

Fig. 1 The original $K$-plet representation. Here, $\left(r_{i}, \varphi_{i}\right)$ is the polar coordinate of neighbor $i$ with respect to the central minutia, $m_{c} . \theta_{i}$ is the relative angle of neighbor $i$ with respect to $m_{c}$. (Illustration inspired by Chikkerur et al. (2005)

3. One of the point sets might only be a subset of the other. Noisy images might have both removed feature points and introduced new false ones.

4. Point pattern problems often involve huge datasets which leads to a necessity for a fast and effective matching strategy.

Although this paper focuses on point pattern matching, there are other related techniques to match sets of points to each other. A common example is the $3 \mathrm{D}$ shape matching algorithm Iterative Closest Point (ICP) proposed by Besl and McKay (1992) and Chen and Medioni (1992).

\section{$2.2 \mathrm{~K}$-plet representation}

This article presents a feature point neighborhood representation called K-plets, which was originally proposed as a fingerprint descriptor by Chikkerur et al. (2005). The original paper, with its nomenclature, is shortly presented here as an introduction to the subject.

A $K$-plet describes the geometry of a local neighborhood around a certain fingerprint minutia (Figure 1). It consists of a central minutia, $m_{c}$, and $K$ neighboring minutiae $\left\{m_{1}, m_{2}, \ldots, m_{K}\right\}$. Each neighborhood minutia is described by its distance, $r$, to the central minutia, the angle, $\varphi$, of the line connecting the central minutia and the neighbor, and the relative orientation of the neighboring minutiae compared to that of the central minutia, $\theta$.

Complete rotational invariance is obtained by rotating the $K$-plet to align the direction of the central minutia, $\theta_{c}$, with the $x$-axis.

The neighbors of each $K$-plet are put in a sequence ordered by increasing radial distance. Later, the matching is carried out by comparing two ordered sequences using a string alignment algorithm. This type of matching strategy handles the possible problem of one or a few missing or added neighborhood minutiae in each $K$-plet. However, this added functionality of handling missing points increases the complexity and therefore computation time of the matching step. 


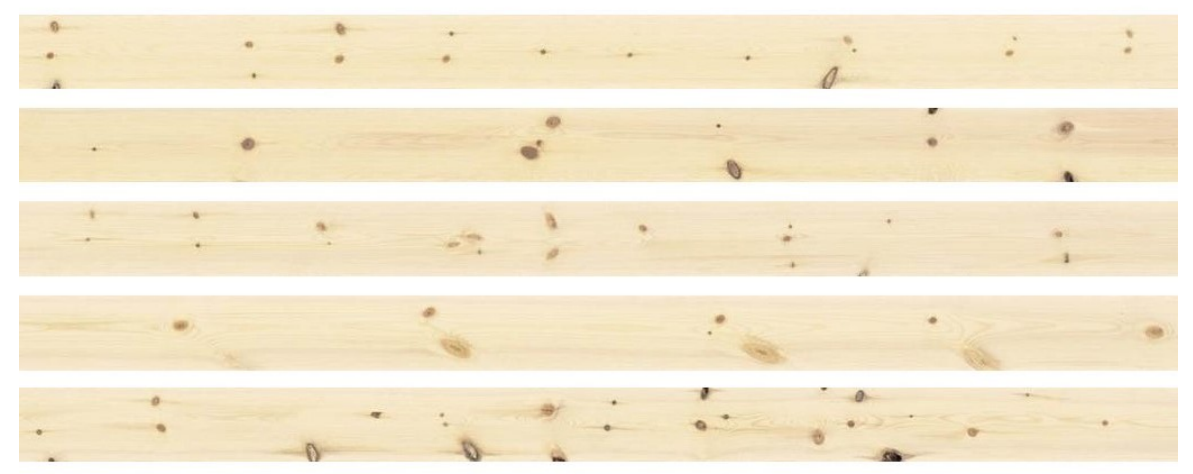

Fig. 2 Example of a few boards from the floorboard dataset showing the variability. (Board lengths have been cropped to fit the page.)

\subsection{Segmenting knots from clearwood}

Knot positions are the main input for the wood fingerprint descriptor that is proposed in this paper. A good segmentation of knots in wood panel images therefore paramount to be able to identify fingerprints. Totally automatic and robust knot segmentation is challenging to achieve using only RGB-images. It can be quite difficult to divide knots and background (clearwood) into two classes since knots can have exactly the same color and brightness as clearwood. Growth rings, reaction wood, pith and other defects can further complicate the segmentation task. However, much work has been done in the field of automatic segmentation of knots on board images (Åstrand 1996; Funck et al. 2003, Todoroki et al. 2010). Modern wood surface scanners often deal with the problem by utilizing both X-ray and the tracheid effect to more accurately detect knots (GoldenEye; WoodEye).

\section{Material and methods}

This section describes how the dataset of panel images that were used for the performance tests were created and how the knot positions were extracted. After that, the $K$-plet descriptors are explained and how they were adapted to work on the panel dataset. Lastly, the descriptor matching procedure is presented and how the performance tests were carried out.

\subsection{Creating a dataset}

A total of 212 simulated panel images were constructed by stitching together board images from an available floorboard dataset. The simulated panel dimensions were chosen to be similar to the formwork panels produced by an industrial partner of the European research project Hol-i-Wood PR. This means panels of $3000 \mathrm{~mm}$ length and four boards $(4 \times 137 \mathrm{~mm}=548 \mathrm{~mm})$ of width.

The floorboard dataset consists of 886 floorboard images from Scots pine (Pinus sylvestris L.) with the dimension $21 \times 137 \mathrm{~mm}$ (Figure 2 that were originally scanned for a customer preference study (Broman et al. 2008; Nyström et al. 2008; Oja et al. 2008). These boards were sawn from 222 logs with top diameter between 201-215 mm which were randomly collected from Bollsta sawmill in central Sweden. 


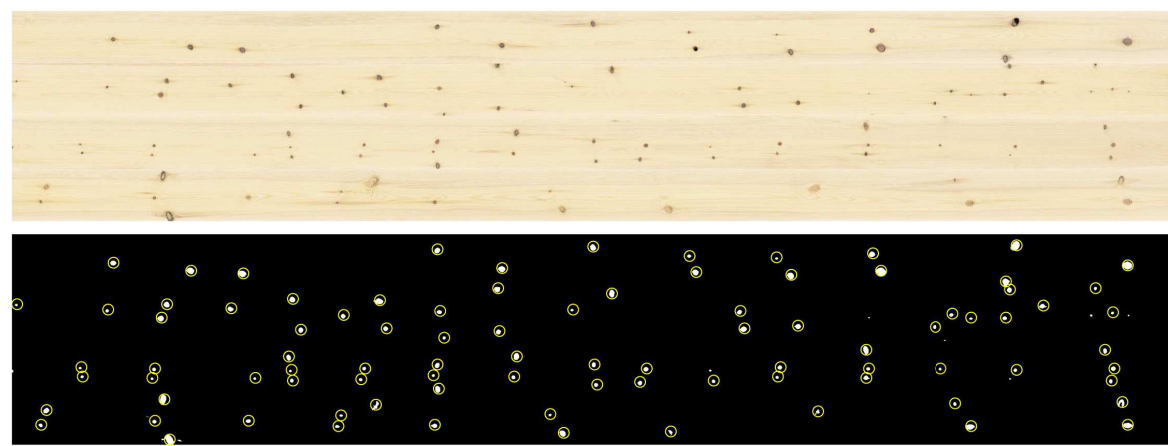

Fig. 3 On top, a simulated floorboard panel created by stitching together four floorboards and cutting off their lengths at $3000 \mathrm{~mm}$. The lower image shows the thresholded version of the same panel, where the segmented knots have been marked by yellow circles.

\subsection{Extraction of knot positions}

The aim of extracting knot positions from the panel dataset was to obtain a database of realistic knot distributions on panels. With such data, the accuracy of the proposed neighborhood descriptors ( $K$-plets) could be tested. For the tests to be relevant, it was not necessary to obtain the exact knot positions from the panel dataset as long as the knot distributions were realistic.

To extract knot positions, a semi-automatic approach was adopted. First, an automatic segmentation of knots was made as described in the list below, and then the segmented image and knot centroids were displayed on screen as seen in Figure 3. If the positions were deemed approximately correct by manual inspection, the centroid positions, $(x, y)$, were saved. The exact rejection criteria in the manual inspection are not crucial for this study; it suffices to say that outliers were removed in this step. Below follows a detailed description of the knot centroid extraction procedure, which was conducted in MATLAB:

1. Pick 4 board images from the dataset. Crop the board images to $3000 \mathrm{~mm}$ length, stitch them together to a panel and convert it to grayscale.

2. Smooth the image using a $5 \times 5 \mathrm{~mm}$ averaging filter to suppress growth rings.

3. Perform an edge detection with a morphological black top-hat filtering (rolling ball filter) using a circular structuring element with radius $12 \mathrm{~mm}$. Knots are accentuated by this procedure while regions of compression wood, which have similar appearance as uneven lighting, are suppressed. The transformation of image $I$ is defined as: $T_{S E}(I)=I \bullet S E-I$, where $\bullet$ is a closing operation and $S E$ is a grayscale structuring element.

4. Threshold the resulting image into two classes using Otsu's method (Otsu 1979). (The method tries to separate an image into two classes, black and white, while minimizing the intra-class variance.)

5. Fill holes in the logical image, $B W$, using the MATLAB command: $\operatorname{imfill}\left(B W\right.$, 'holes' $\left.^{\prime}\right)$. This function performs hole filling by morphological reconstruction (Gonzalez and Woods 2008, p. $660)$.

6. Remove small or thin regions in the image by aid of the MATLAB function regionprops (MathWorks MATLAB a). A region is considered small or thin if the area is less than $36 \mathrm{~mm}^{2}$ or the eccentricity is higher than 0.98 . These values were chosen since a majority of such regions in the logical images were unlikely to be knots. This fact was observed by manual inspection. 


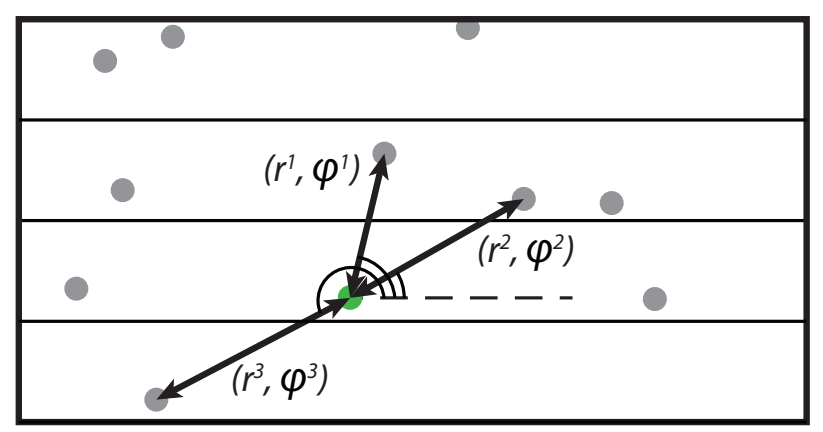

Fig. 4 The neighborhood descriptor for $K=3$ visualized on a surface with gray circles representing knots. Each of the $K$ neighboring knots has a distance $r^{j}$ at an angle $\varphi^{j}$ to the center knot.

\subsection{Neighborhood descriptors ( $K$-plets)}

The $K$-plet neighborhood descriptor in our implementation is similar to the one used by Chikkerur et al. (2005). An exception is that there is no orientation included for our minutiae, i.e., knots. This parameter has been neglected in this work to keep a low computational complexity and to lower the requirements of a knot segmentation algorithm.

After a knot detection algorithm has obtained the centroids of the knots on a wood piece, the problem boils down to matching points between images. $K$-plets describe local geometric relationships between points and can thus also be used for wood fingerprint recognition.

The distances and angles from every knot to $K$ neighboring knots were picked as descriptors of knot neighborhoods. See Figure 4 for an example using three knot neighbors. Note that, in our case the neighborhoods often span over several boards on the panels, but there is no principle reason why the proposed method would not also work on single boards.

The neighborhood $K$-plets' distances and angles for one panel can be stored as rows in a matrix for each wood piece according to:

$$
P=\left[\begin{array}{cccc|cccc}
r_{1}^{1} & r_{1}^{2} & \ldots & r_{1}^{K} & \varphi_{1}^{1} & \varphi_{1}^{2} & \ldots & \varphi_{1}^{K} \\
r_{2}^{1} & r_{2}^{2} & \ldots & r_{2}^{K} & \varphi_{2}^{1} & \varphi_{2}^{2} & \ldots & \varphi_{2}^{K} \\
\vdots & \vdots & & \vdots & \vdots & \vdots & & \vdots \\
r_{m}^{1} & r_{m}^{2} & \ldots & r_{m}^{K} & \varphi_{m}^{1} & \varphi_{m}^{2} & \ldots & \varphi_{m}^{K}
\end{array}\right]
$$

where the neighbors are sorted with ascending value of $r$, i.e., $r_{i}^{1}$, and $\varphi_{i}^{1}$ correspond to the closest neighbor to knot $i . m$ is both the number of knots and number of $K$-plets on the panel.

\subsubsection{Scale- and rotational invariance}

Scale- and rotational invariance is obtained by computing ratios between the radial distances and differences between the angles within each $K$-plet. The matrix $P$ in Equation 1 is modified as

$$
P^{*}=\left[\begin{array}{cccc|cccc}
r_{1}^{(2 / 1)} & r_{1}^{(3 / 1)} & \ldots & r_{1}^{(K / 1)} & \varphi_{1}^{(2-1)} & \varphi_{1}^{(3-1)} & \ldots & \varphi_{1}^{(K-1)} \\
r_{2}^{(2 / 1)} & r_{2}^{(3 / 1)} & \ldots & r_{2}^{(K / 1)} & \varphi_{2}^{(2-1)} & \varphi_{2}^{(3-1)} & \ldots & \varphi_{2}^{(K-1)} \\
\vdots & \vdots & & \vdots & \vdots & \vdots & & \vdots \\
r_{m}^{(2 / 1)} & r_{m}^{(3 / 1)} & \ldots & r_{m}^{(K / 1)} & \varphi_{m}^{(2-1)} & \varphi_{m}^{(3-1)} & \ldots & \varphi_{m}^{(K-1)}
\end{array}\right]
$$

where $P^{*}$ is of size $m \times(2 K-2)$ and each row corresponds to a $K$-plet. In the matrix, $r_{i}^{(j / 1)}=r_{i}^{j} / r_{i}^{1}$ and $\varphi_{i}^{(j-1)} \in[0, \pi]$ is the closest angle between knots $j$ and 1 for the $i$ :th $K$-plet. 


\subsubsection{Normalization of descriptor data}

The $K$-plet column values need to be normalized in order for all distance ratios and angles to have the same weight in the identification. All $P^{*}$ matrices for the query and database panels were normalized column-wise according to

$$
P^{*} \longrightarrow\left[\frac{P_{1}^{*}-\mu_{1}}{\sigma_{1}}, \frac{P_{2}^{*}-\mu_{2}}{\sigma_{2}}, \ldots, \frac{P_{2 K-2}^{*}-\mu_{2 K-2}}{\sigma_{2 K-2}}\right]
$$

where $P_{i}^{*}$ is a column vector of length $m . \mu_{i}$ and $\sigma_{i}$ are the mean and standard deviation of $P_{i}^{*}$.

\subsection{Matching of descriptors}

To identify a query panel in a database of panels, the $K$-plets of the query panel are compared to the $K$-plets of each database panel. The best matching database panel is obtained by counting the number of matching $K$-plets, $N$, for each database panel and evaluating which panel gave the highest value. To illustrate this procedure, we will describe how $N$ is calculated when matching $K$-plets from a query panel, $Q$, to those of a specific panel in the database, $D$. This calculation is done in two steps, where the first is to obtain a set of probable matches, and the second is to refine that selection by removing erroneous matches.

\subsubsection{Sum of squared differences}

The quality of the match between two $K$-plets is calculated as the sum of squared differences (SSD) of the elements (squared Euclidean distance). A perfect match will have an SSD of 0, while a bad match will have a relatively high SSD. The SSD is calculated for all possible $K$-plet pairs between $Q$ and $D$. All matches that have an SSD $<\lambda$ are extracted and subject to further processing as described in Section 3.4 .2 We denote this set of matching $K$-plets as $\Lambda$. The optimal threshold, $\lambda$, for our dataset was computed in the matching performance test.

\subsubsection{Removal of erroneous matches}

MATLAB's geometric transform estimator (GTE) was utilized on the centroids of the matching $K$-plet pairs in $\Lambda$ to remove outlier matches and thus improving the panel matching accuracy. The number of inliers, $N$, after applying the GTE was used as a measure of how well a database panel matches a query panel. The best matching panel in the database was the one with the highest value of $N$.

The GTE uses Random Sample Consensus (RANSAC, (Fischler and Bolles 1981)) to enable completion of the estimation within a given time frame. We assume only simple geometric differences between query and database panel images, i.e., a similarity transformation consisting of rotation, scale and translation as seen in Figure 5. The GTE will iterate and randomly select, in this case, two centroid pairs to compute a transformation matrix that best fits the input points. The success of estimating a correct geometric transformation using RANSAC heavily depends on the percentage of correctly matched point pairs given as input (MathWorks MATLAB b). To obtain good results, the percentage of inliers should be kept at the highest level possible. A reasonable inlier percentage is obtained by choosing a suitable $\lambda$-threshold. Another way to improve the panel recognition rate is to increase the number of random samplings (iterations). Herein lies a trade-off between better results and computation time. The settings for the GTE were in this study chosen as in Table 1. 


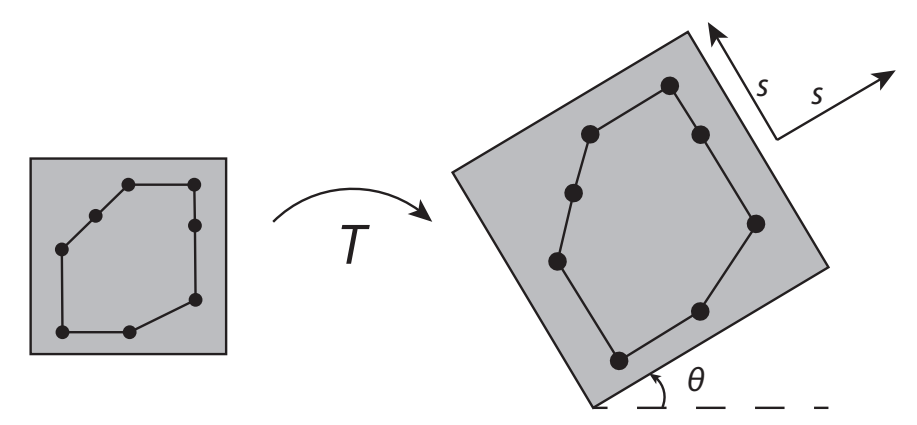

Fig. 5 The similarity transformation, $T$, where $\theta$ is the rotation and $s$ is an isotropic scale factor.

Table 1 Settings for the geometric transform estimator used in our tests.

\begin{tabular}{lr}
\hline Parameter & Setting \\
\hline Method & 'Random Sample Consensus (RANSAC)' \\
ExcludeOutliers & true \\
Transform & 'Nonreflective similarity' \\
PixelDistanceThreshold & 100 \\
NumRandomSamplings & 1000 \\
\hline
\end{tabular}

\subsection{Matching performance test}

Since the proposed matching algorithm relies upon knot centroids, it is important to test how the knot segmentation precision affects the panel recognition rate. To evaluate the effect of knot precision, three error levels were added to the detected knot centroid positions. These errors were normally distributed with zero mean and standard deviations $\sigma_{\epsilon}=[5,8,11] \mathrm{mm}$. Hence, three databases of $K$-plets, $D_{\sigma}$, were created which corresponded to a specific knot positional error level, measured in Euclidean distance.

The query datasets were created similarly to $D_{\sigma}$, but also a portion, $p_{\epsilon}$, of the knots were now removed. With three error levels in knot position, $\sigma_{\epsilon}=[5,8,11] \mathrm{mm}$, and four distinct knot removal portions, $p_{\epsilon}=[0,5,10,15] \%$, twelve query datasets, $Q_{\sigma, p}$, with specific error levels were created.

The twelve query datasets were matched to the database having the same error level in knot position. For example, the panels in query datasets $\left\{Q_{5,0}, Q_{5,5}, Q_{5,10}, Q_{5,15}\right\}$ were matched against the panels in database $D_{5}$. Hence, a total of twelve test cases were studied. The matching accuracy was calculated as the percentage of correctly matched panels. Thus, twelve accuracy values were calculated, which each corresponds to a specific error level.

To get a better understanding of the impact of the value $K$, several $K$-values were evaluated: $K \in\{4,6,8,10\}$. The previously described procedure of validation was used on each choice of $K$-values.

The mean computational time of matching one query panel to a database panel was calculated. This was done in MATLAB R2013b by measuring the time the matching algorithm needed to match all 212 query panels to all 212 database panels twelve times (once for each error level). In other words, this means a total of $212 \times 212 \times 12$ times. The measurement was done with the $K$ and $\lambda$-value which gave the best accuracy. A laptop of model HP EliteBook 8560w with Intel Core i7-2670QM processor at $2.20 \mathrm{GHz}, 64$-bit Windows 7 operating system and 8 GB RAM was used. 


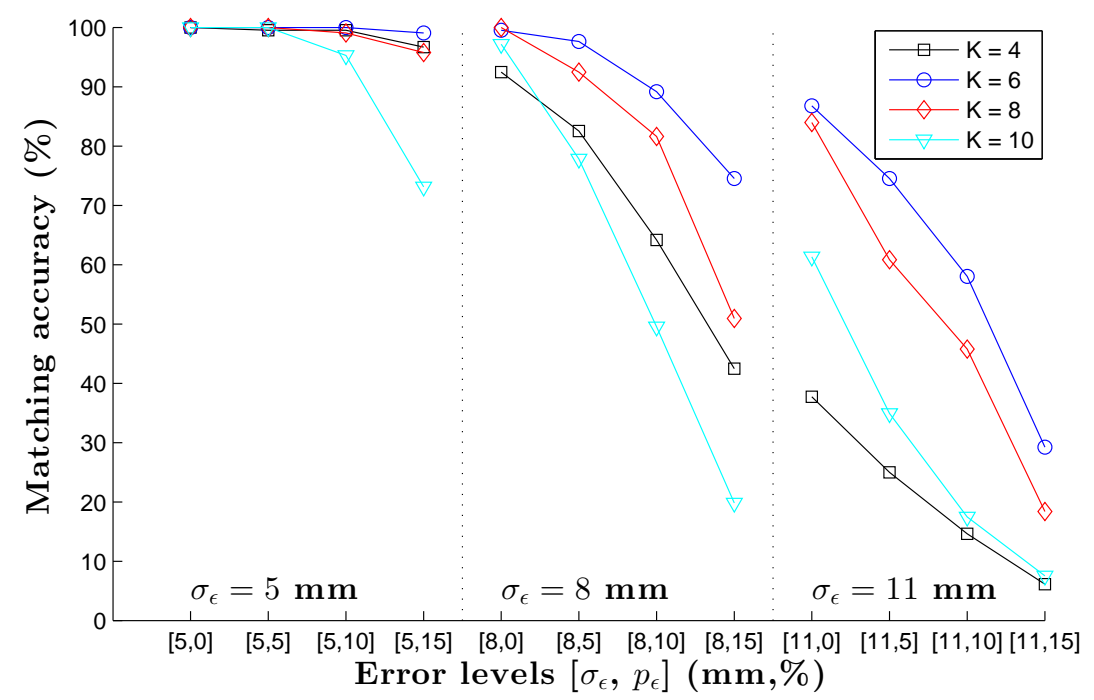

Fig. 6 The matching accuracy for different number of knot neighbors, $K$, knot positional error level, $\sigma_{\epsilon}$, and percentage of removed knots, $p_{\epsilon}$.

\section{Results}

The results of the matching accuracy tests described in Section 3.5 are shown in Figure 6. From this figure it is obvious that $K=6$ is the optimal value in this study. To obtain an accuracy of at least $99 \%$ with $K=6$, the positional error standard deviation must not exceed $5 \mathrm{~mm}$ whilst detecting at least $85 \%$ of the knots in one set. If all knots can be successfully detected, then the knot positional error is allowed to be $8 \mathrm{~mm}$. Detailed results with $K=6$ are shown in Table 2 The results in this table highlight the importance of maintaining a low knot positional error.

The SSD threshold for accepting possible matches was set to $\lambda=1.4$. This specific threshold was chosen since it resulted in the best overall performance of the algorithm. However, test runs showed that the performance of the algorithm was quite stable even when changing the parameter $\lambda$.

When using $K=6$ and $\lambda=1.4$, the tests of computational time showed that the average time of matching two panels to each other was $3.65 \mathrm{~ms}$. Hence, if the database would contain 1000 panels, the average computational time for matching a query panel to such a database would be $3.65 \mathrm{~s}$ using the same hardware setup and MATLAB implementation. This may at least give a hint of what times can be expected.

Table 2 Accuracy of the matching algorithm with $K=6$ and $\lambda=1.4$.

\begin{tabular}{lcccccccccccc}
\hline Accuracy (\%) & 100 & 100 & 100 & 99.5 & 100 & 96.7 & 89.2 & 74.1 & 87.3 & 73.6 & 58.5 & 28.3 \\
\hline Positional error (mm) & 5 & 5 & 5 & 5 & 8 & 8 & 8 & 8 & 11 & 11 & 11 & 11 \\
Removed knots (\%) & 0 & 5 & 10 & 15 & 0 & 5 & 10 & 15 & 0 & 5 & 10 & 15 \\
\hline
\end{tabular}




\section{Discussion}

The proposed method of using $K$-plet descriptors, SSD matcher and geometrical transform estimator, is scale- and rotationally invariant. This fact renders the method useful in more applications than just panel matching with well-defined orientation and scale. For example, if a mobile robot with an attached vision system is used, the robot has the possibility to recognize wood products at various distances and orientations.

To work in wood manufacture, methods for recognition of wood products often need to handle possible division of the wood. The proposed method handles this scenario to a certain extent. However, the wood pieces must then contain enough knots to be well defined with $K$-plets. Furthermore, the $\mu$ and $\sigma$ of the piece can not change too much.

For matching algorithms to be used in practice they need high enough matching accuracy. The necessary accuracy depends on the application but in our specific case of matching panels it should be higher than $99 \%$. Our results show that this is possible with the proposed method if the error in knot position has a standard deviation of $5 \mathrm{~mm}$ or less. It is important for the accuracy that the knot segmentation is consistent on both the database image and the corresponding query image. The image acquisition needs to be similar enough so that the image quality does not affect the knot segmentation. Aside from errors related to knot segmentation, the accuracy is affected by the size of the database and the frequency of knots. A smaller database would likely increase the accuracy since it would decrease the search space in the matching step. This would also reduce the total computational time which increases linearly with database size. The panels used in this study had a relatively high number of knots, but if the algorithm is used on panels with just a handful of knots, it would likely be rather inaccurate. How many knots that are needed has not been investigated and such a threshold is difficult to guess. However, there is no reason to expect that the algorithm would not work if only half the amount of knots were available compared to the panels used in this study.

Funck et al. (2003) evaluated several wood defect detection algorithms on color images of Douglas fir (Pseudotsuga menziesii (Mirb.) Franco) veneer sheets. The color images used in that study had a resolution of $1.7 \mathrm{~mm} /$ pixel compared to our color images with $1 \mathrm{~mm} /$ pixel. In their tests, many of the algorithms had a mean error of less than $5 \mathrm{~mm}$ when calculating knot centroid position. This indicates that our proposed feature matching algorithm is feasible for industrial applications. However, in the current study, the panels used were constructed from boards which are cut in a different way than veneer sheets. Knot shape is approximately circular on veneer sheets, but on boards they have more diverse shapes which makes knots more difficult to segment. The results from Funck et al. (2003) still give an indication of the expected accuracy in knot position measurements on wooden boards. Knot segmentation algorithms are therefore likely sufficiently accurate for applications using our matching algorithm.

There are several ways to improve this algorithm further. In this paper, the $K$-plets consist of knot centroids, but they could also incorporate knot shape and type, as well as size and orientation relative to the other knots in the $K$-plet. This adds other requirements to a knot segmentation algorithm, but with extra information it is reasonable to expect that the requirements of knot centroid precision would decrease.

\section{Conclusions}

- This paper presents an algorithm for matching sawn wood products. The proposed method opens up possibilities to automate and individualize the processing of products. It becomes possible to trace and steer good or bad raw material properties through a process chain despite a disorganized product flow and several processing steps that slightly alters the appearance of the product. 
- The method is scale- and rotationally invariant, which means that an autonomous robot could recognize the product at various distances and the product does not have to be oriented in any special way.

- The proposed algorithm uses a novel approach based on internal knot structure. For the Scots pine panels used in this study, the algorithm was successful, as should be the case for panels of other wood species that contain a similar frequency of knots.

- Our algorithm uses knot positions; consequently the knot segmentation accuracy is very important. According to previous publications on knot segmentation, the proposed matching algorithm should be good enough for industrial applications as long as the input images are without geometric distortions.

Acknowledgements This work has been part of the Hol-i-Wood Patching Robot project and received funding from the European Union's Seventh Framework Programme (FP7/2007-2013) under grant agreement no. 284573. The Hol-i-Wood PR is a collaboration between Luleå University of Technology (LTU), TU Wien and TU München, as well as industrial partners; MiCROTEC, Springer, TTTech and LIP-BLED.

\section{References}

Åstrand, E., 1996. Automatic Inspection of Sawn Wood. Linköping Studies in Science and Technology, Linköping University.

Besl, P.J., McKay, N.D., 1992. A method for registration of 3-d shapes. IEEE T Pattern Anal 14, 239-256.

Broman, N.O., Nyström, J., Oja, J., 2008. Modelling the connection between industrially measured raw material properties and end user preferences. Part 2 - Results from preference studies, in: Proceedings of the IUFRO Working Party 5.01.04, Joensuu, Finland.

Brown, M., Lowe, D.G., 2007. Automatic panoramic image stitching using invariant features. Int. J. Comput. Vision 74, 59-73.

Chang, S.H., Cheng, F.H., Hsu, W.H., Wu, G.Z., 1997. Fast algorithm for point pattern matching: Invariant to translations, rotations and scale changes. Pattern Recognition 30, $311-320$.

Chen, Y., Medioni, G., 1992. Object modelling by registration of multiple range images. Image Vision Comput. 10, 145-155.

Chikkerur, S., Cartwright, A., Govindaraju, V., 2005. K-plet and coupled bfs: A graph based fingerprint representation and matching algorithm, in: Zhang, D., Jain, A. (Eds.), Advances in Biometrics. Springer Berlin Heidelberg. volume 3832 of Lecture Notes in Computer Science, pp. 309-315.

Dykstra, D.P., Kuru, G., Taylor, R., Nussbaum, R., Magrath, W.B., Story, J., 2002. Technologies for Wood Tracking, In: Environmental and Social Development East Asia and Pacific Region Discussion Paper. Technical Report. World Bank.

Fischler, M.A., Bolles, R.C., 1981. Random sample consensus: A paradigm for model fitting with applications to image analysis and automated cartography. Comm ACM 24, 381-395.

Flodin, J., 2009. KAP - Kontinuerlig Automatisk Provsågning. Technical Report. WoodCenter North. In Swedish.

Funck, J., Zhong, Y., Butler, D., Brunner, C., Forrer, J., 2003. Image segmentation algorithms applied to wood defect detection. Computers and Electronics in Agriculture 41, 157 - 179.

GoldenEye, . Microtec GmbH, http://www.microtec.eu.

Gonzalez, R., Woods, R., 2008. Digital Image Processing (3rd Edition). Pearson Prentice Hall.

Gruen, A., Akca, D., 2005. Least squares 3d surface and curve matching. ISPRS J Photogramm $59,151-174$.

Jain, A.K., Flynn, P., Ross, A.A., 2007. Handbook of Biometrics. Springer-Verlag New York, Inc., Secaucus, NJ, USA. 
MathWorks MATLAB, 2013a. Image Processing Toolbox: 2013b, The MathWorks Inc., Natick, Massachusetts, United States.

MathWorks MATLAB, 2013b. Computer Vision System Toolbox: 2013b, The MathWorks Inc., Natick, Massachusetts, United States.

Nyström, J., Oja, J., Broman, N.O., 2008. Modelling the connection between industrially measured raw material properties and end user preferences. Part 1 - The simulation tool, in: Proceedings of the IUFRO Working Party 5.01.04, Joensuu, Finland.

Oja, J., Broman, N.O., Nyström, J., 2008. Modelling the connection between industrially measured raw material properties and end user preferences. Part 3 - Optimizing the industrial production, in: Proceedings of the IUFRO Working Party 5.01.04, Joensuu, Finland.

Otsu, N., 1979. A Threshold Selection Method from Gray-level Histograms. IEEE Transactions on Systems, Man and Cybernetics 9, 62-66.

Spratling, B.B., Mortari, D., 2009. A survey on star identification algorithms. Algorithms 2, 93-107.

Todoroki, C., Lowell, E., Dykstra, D., 2010. Automated knot detection with visual post-processing of douglas-fir veneer images. Computers and Electronics in Agriculture 70, $163-171$.

Uusijärvi, R., 2000. Automatic tracking of wood. Ph.D. thesis. Kungliga Tekniska Högskolan. Institutionen för produktionssystem.

WoodEye, . Innovativ Vision AB, http://www.woodeye.se. 